CREAT. MATH. INFORM.

Volume 29 (2020), No. 2,

Pages 145 - 152
Online version at https : //creative-mathematics. cunbm. utcluj.ro/ Print Edition: ISSN 1584 - 286X; Online Edition: ISSN 1843 - 441X

DOI: https://doi.org/10.37193/CMI.2020.02.05

\title{
Nonlinear elliptic anisotropic problem involving non-local boundary conditions with variable exponent and graph data
}

\author{
ADAMA KabORE and StANislas OUARO
}

ABSTRACT. We study a nonlinear elliptic anisotropic problem involving non-local conditions. We also consider variable exponent and general maximal monotone graph datum at the boundary. We prove the existence and uniqueness of weak solution to the problem.

\section{INTRODUCTION AND ASSUMPTIONS}

Let $\Omega$ be a bounded domain in $\mathbb{R}^{N}(N \geq 2)$ such that $\partial \Omega$ is Lipschitz and $\partial \Omega=\Gamma_{D} \cup \Gamma_{N e}$ with $\Gamma_{D} \cap \Gamma_{N e}=\emptyset$ and $\operatorname{dist}\left(\Gamma_{D}, \Gamma_{N e}\right)>0$. Our aim is to study the following problem

$$
S_{f, d}^{\rho} \begin{cases}-\sum_{i=1}^{N} \frac{\partial}{\partial x_{i}} a_{i}\left(x, \frac{\partial}{\partial x_{i}} u\right)+|u|^{P_{M}(x)-2} u=f & \text { in } \Omega \\ u=0 & \text { on } \Gamma_{D} \\ \rho(u)+\sum_{i=1}^{N} \int_{\Gamma_{N e}} a_{i}\left(x, \frac{\partial}{\partial x_{i}} u\right) \eta_{i} \ni d \\ u \equiv \text { constant } & \text { on } \Gamma_{N e},\end{cases}
$$

where the right-hand side $f \in L^{\infty}(\Omega)$ and $\eta_{i}, i \in\{1, \ldots, N\}$ are the components of the outer normal unit vector, $\rho$ a maximal monotone graph on $\mathbb{R}$ such that

$$
D(\rho)=\mathbb{R}, \operatorname{Im}(\rho)=\mathbb{R} \text { and } 0 \in \rho(0) .
$$

For any $\Omega \subset \mathbb{R}^{N}$, we set

$$
C_{+}(\bar{\Omega})=\left\{h \in C(\bar{\Omega}): \inf _{x \in \Omega} h(x)>1\right\},
$$

and we denote

$$
h^{+}=\sup _{x \in \Omega} h(x), h^{-}=\inf _{x \in \Omega} h(x) .
$$

We consider the exponents, $\vec{p}():. \bar{\Omega} \rightarrow \mathbb{R}^{N}$ such that $\vec{p}()=.\left(p_{1}(),. \ldots, p_{N}().\right)$ with $p_{i} \in$ $C_{+}(\bar{\Omega})$ for every $i \in\{1, \ldots, N\}$ and for all $x \in \bar{\Omega}$. We put $p_{M}(x)=\max \left\{p_{1}(x), \ldots, p_{N}(x)\right\}$ and $p_{m}(x)=\min \left\{p_{1}(x), \ldots, p_{N}(x)\right\}$.

We assume that for $i=1, \ldots, N$, the function $a_{i}: \Omega \times \mathbb{R} \rightarrow \mathbb{R}$ is Carathéodory and satisfies the following conditions.

- $\left(H_{1}\right): a_{i}(x, \xi)$ is the continuous derivative with respect to $\xi$ of the mapping $A_{i}=$ $A_{i}(x, \xi)$, that is, $a_{i}(x, \xi)=\frac{\partial}{\partial \xi} A_{i}(x, \xi)$ such that the following equality holds.

$$
A_{i}(x, 0)=0,
$$

Received: 12.11.2019. In revised form: 04.09.2020. Accepted: 11.09.2020

2010 Mathematics Subject Classification. 35J05, 35J25, 35J60, $35 \mathrm{~J} 66$.

Key words and phrases. Maximal monotone graph, non-local boundary conditions, variable exponent, Leray-Lions operator.

Corresponding author: Adama Kabore; ouaro@yahoo.fr 
for almost every $x \in \Omega$.

- $\left(H_{2}\right)$ : There exists a positive constant $C_{1}$ such that

$$
\left|a_{i}(x, \xi)\right| \leq C_{1}\left(j_{i}(x)+|\xi|^{p_{i}(x)-1}\right),
$$

for almost every $x \in \Omega$ and for every $\xi \in \mathbb{R}$, where $j_{i}$ is a non-negative function in $L^{p_{i}^{\prime}(.)}(\Omega)$, with $\frac{1}{p_{i}(x)}+\frac{1}{p_{i}^{\prime}(x)}=1$.

- $\left(H_{3}\right)$ : There exists a positive constant $C_{2}$ such that

$$
\left(a_{i}(x, \xi)-a_{i}(x, \eta)\right) \cdot(\xi-\eta) \geq \begin{cases}C_{2}|\xi-\eta|^{p_{i}(x)} & \text { if }|\xi-\eta| \geq 1 \\ C_{2}|\xi-\eta|^{p_{i}^{-}} & \text {if }|\xi-\eta|<1\end{cases}
$$

for almost every $x \in \Omega$ and for every $\xi, \eta \in \mathbb{R}^{N}$, with $\xi \neq \eta$.

- $\left(H_{4}\right)$ : For almost every $x \in \Omega$ and for every $\xi \in \mathbb{R}^{N}$,

$$
|\xi|^{p_{i}(x)} \leq a_{i}(x, \xi) \cdot \xi \leq p_{i}(x) A_{i}(x, \xi) .
$$

Non-local boundary value problems of various kinds for partial differential equations are of great interest by now in several fields of application. In a typical non-local problem, the partial differential equation (resp. boundary conditions) for an unknown function $u$ at any point in a domain $\Omega$ involves not only the local behavior of $u$ in a neighborhood of that point but also the non-local behavior of $u$ elsewhere in $\Omega$. For example, at any point in $\Omega$ the partial differential equation and/or the boundary conditions may contains integrals of the unknown $u$ over parts of $\Omega$, values of $u$ elsewhere in $D$ or, generally speaking, some non-local operator on $u$. Beside the mathematical interest of nonlocal conditions, it seems that this type of boundary condition appears in petroleum engineering model for well modeling in a $3 D$ stratified petroleum reservoir with arbitrary geometry (see [3] and [4]).

\section{PRELIMINARY AND MAIN RESULT}

This part is related to anisotropic Lebesgue and Sobolev spaces with variable exponent, some of their properties (for more details see [6] and [7]) and the main result of the paper. Given a measurable function $p():. \Omega \rightarrow[1, \infty)$. We define the Lebesgue space with variable exponent $L^{p(.)}(\Omega)$ as the set of all measurable functions $u: \Omega \rightarrow \mathbb{R}$ for which the convex modular

$$
\rho_{p(.)}(u):=\int_{\Omega}|u|^{p(x)} d x
$$

is finite.

If the exponent is bounded, i.e, if $p_{+}<\infty$, then the expression

$$
|u|_{p(.)}:=\inf \left\{\lambda>0: \rho_{p(.)}\left(\frac{u}{\lambda}\right) \leq 1\right\}
$$

defines a norm in $L^{p(.)}(\Omega)$, called the Luxembourg norm. The space $\left(L^{p(.)}(\Omega),|\cdot|_{p(.)}\right)$ is a separable Banach space. Then, $L^{p(.)}(\Omega)$ is uniformly convex, hence reflexive and its dual space is isomorphic to $L^{p^{\prime}(.)}(\Omega)$, where $\frac{1}{p(x)}+\frac{1}{p^{\prime}(x)}=1$, for all $x \in \Omega$.

The anisotropic variable exponent Sobolev space $W^{1, \vec{p}(.)}(\Omega)$ is defined as follow.

$$
W^{1, \vec{p}(.)}(\Omega):=\left\{u \in L^{p_{M}(.)}(\Omega): \frac{\partial u}{\partial x_{i}} \in L^{p_{i}(.)}(\Omega), \text { for all } i \in\{1, \ldots, N\}\right\} .
$$


Endowed with the norm

$$
\|u\|_{\vec{p}(.)}:=|u|_{p_{M}(.)}+\sum_{i=1}^{N}\left|\frac{\partial u}{\partial x_{i}}\right|_{p_{i}(.)},
$$

the space $\left(W^{1, \vec{p}(.)}(\Omega),\|\cdot\|_{\vec{p}(.)}\right)$ is a reflexive Banach space (see [7], Theorem 2.1 and Theorem 2.2).

As consequence, we have the following.

Let us introduce the following notation:

$$
\vec{p}_{-}=\left(p_{1}^{-}, \ldots, p_{N}^{-}\right) .
$$

In the sequel, we consider the following spaces.

$$
W_{D}^{1, \vec{p}(.)}(\Omega)=\left\{\xi \in W^{1, \vec{p}(.)}(\Omega): \xi=0 \text { on } \Gamma_{D}\right\}
$$

and

$$
W_{N e}^{1, \vec{p}(.)}(\Omega)=\left\{\xi \in W_{D}^{1, \vec{p}(.)}(\Omega): \xi \equiv \text { constant on } \Gamma_{N e}\right\} .
$$

For any $v \in W_{N e}^{1, \vec{p}(.)}(\Omega)$, we set $v_{N}=v_{N e}:=\left.v\right|_{\Gamma_{N e}}$.

The concept of solution for $S_{f, d}^{\rho}$ is given as follows.

Definition 2.1. A solution of $S_{f, d}^{\rho}$ is a couple $(u, v) \in W_{N e}^{1, \vec{p}(.)}(\Omega) \times \mathbb{R}$ satisfying

$$
\left\{\begin{array}{l}
w=|u|^{p_{M}(x)-2} u \text { a.e. in } \Omega, v \in \rho\left(u_{N}\right) \\
\varphi \in W_{N e}^{1, \vec{p}(.)}(\Omega) \cap L^{\infty}(\Omega) \\
\int_{\Omega}\left(\sum_{i=1}^{N} a_{i}\left(x, \frac{\partial}{\partial x_{i}} u\right) \frac{\partial}{\partial x_{i}} \varphi\right) d x+\int_{\Omega} w \varphi d x=\int_{\Omega} f \varphi d x+(d-v) \varphi_{N e} .
\end{array}\right.
$$

Our main result in this paper is the following theorem.

Theorem 2.1. For any $(f, d) \in L^{\infty}(\Omega) \times \mathbb{R}$, the problem $S_{f, d}^{\rho}$ admits at least one solution $(u, v)$ in the sense of Definition 2.1. Moreover if $\left(u_{1}, v_{1}\right)$ and $\left(u_{2}, v_{2}\right)$ are two solutions of $S_{f, d}^{\rho}$, then

$$
\left(v_{1}-v_{2}\right)^{+}+\int_{\Omega}\left(w_{1}-w_{2}\right)^{+} d x \leq \int_{\Omega}\left(f_{1}-f_{2}\right)^{+} d x+\left(d_{1}-d_{2}\right)^{+},
$$

where $w_{1}=\left|u_{1}\right|^{p_{M}(x)-2} u_{1}$ and $w_{2}=\left|u_{2}\right|^{p_{M}(x)-2} u_{2}$.

\section{PROOF OF THE MAIN RESUlT}

The proof of the main result is done in three steps.

Step 1: Approximated problem for continuous functions. We assume that $\rho$ is a continuous, non-decreasing and onto function on $\mathbb{R}$ such that

$$
\rho(0)=0 .
$$

We define a new bounded domain $\tilde{\Omega}$ in $\mathbb{R}^{N}$ as follow.

We fix $\theta>0$ and we set $\tilde{\Omega}=\Omega \cup\left\{x \in \mathbb{R}^{N} / \operatorname{dist}\left(x, \Gamma_{N e}\right)<\theta\right\}$. Then, $\partial \tilde{\Omega}=\Gamma_{D} \cup \tilde{\Gamma}_{N e}$ is Lipschitz with $\Gamma_{D} \cap \tilde{\Gamma}_{N e}=\emptyset$.

Let us consider $\tilde{a}_{i}(x, \xi)$ Carathéodory and satisfying (1.5), (1.6), (1.7) and (1.8), for all $x \in$ $\tilde{\Omega}$.

We also consider a function $\tilde{d}$ in $L^{\infty}\left(\tilde{\Gamma}_{N e}\right)$ such that

$$
\int_{\tilde{\Gamma}_{N e}} \tilde{d} d \sigma=d .
$$




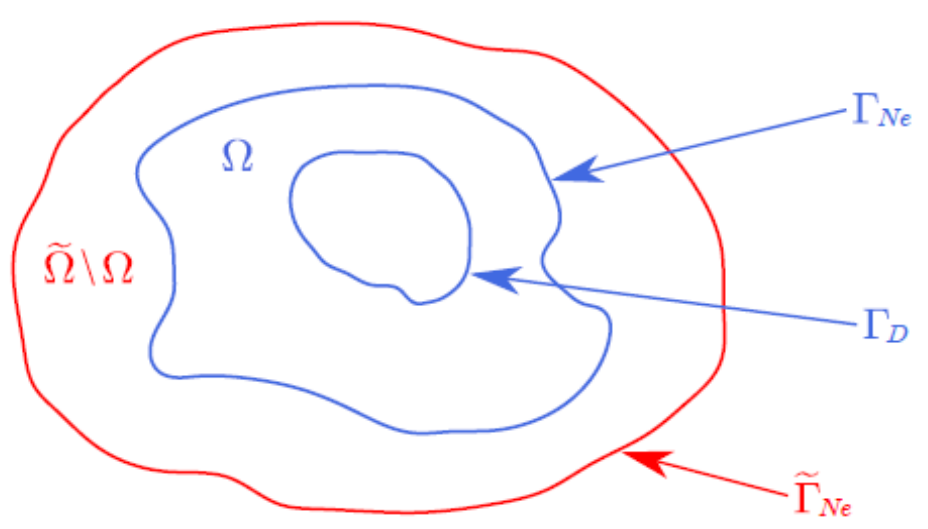

Figure 1: Domains representation

We consider the problem

$$
P(\tilde{\rho}, \tilde{f}, \tilde{d}) \begin{cases}-\sum_{i=1}^{N} \frac{\partial}{\partial x_{i}} \tilde{a}_{i}\left(x, \frac{\partial}{\partial x_{i}} u\right)+|u|^{P_{M}(x)-2} u \chi_{\Omega}(x)=\tilde{f} & \text { in } \tilde{\Omega} \\ u=0 & \text { on } \Gamma_{D} \\ \tilde{\rho}(u)+\sum_{i=1}^{N} \tilde{a}_{i}\left(x, \frac{\partial}{\partial x_{i}} u\right) \eta_{i}=\tilde{d} & \text { on } \tilde{\Gamma}_{N e}\end{cases}
$$

where the function $\tilde{\rho}$ is defined as follow.

- $\tilde{\rho}(s)=\frac{1}{\left|\tilde{\Gamma}_{N e}\right|} \rho(s)$, where $\left|\tilde{\Gamma}_{N e}\right|$ denotes the Hausdorff measure of $\tilde{\Gamma}_{N e}$.

- $\tilde{f}(x)=\left(f \chi_{\Omega}\right)(x) \forall x \in \tilde{\Omega}$.

We obviously have $\tilde{f} \in L^{\infty}(\tilde{\Omega}), \tilde{d} \in L^{\infty}\left(\tilde{\Gamma}_{N e}\right)$.

We introduce the following space

$$
W_{D}^{1, \vec{p}(.)}(\tilde{\Omega})=\left\{\xi \in W^{1, \vec{p}(.)}(\tilde{\Omega}): \xi=0 \text { on } \Gamma_{D}\right\}
$$

Definition 3.2. A measurable function $u: \tilde{\Omega} \rightarrow \mathbb{R}$ is a solution to problem $P(\tilde{\rho}, \tilde{f}, \tilde{d})$ if $u \in W_{D}^{1, \vec{p}(.)}(\tilde{\Omega})$ and

$$
\int_{\tilde{\Omega}} \sum_{i=1}^{N} \tilde{a}_{i}\left(x, \frac{\partial}{\partial x_{i}} u\right) \frac{\partial}{\partial x_{i}} \tilde{\varphi} d x+\int_{\Omega}|u|^{P_{M}(x)-2} u \tilde{\varphi} d x=\int_{\Omega} f \tilde{\varphi} d x+\int_{\tilde{\Gamma}_{N e}}(\tilde{d}-\tilde{\rho}(u)) \tilde{\varphi} d \sigma
$$

for any $\tilde{\varphi} \in W_{D}^{1, \vec{p}(.)}(\tilde{\Omega}) \cap L^{\infty}(\Omega)$.

The problem $P(\tilde{\rho}, \tilde{f}, \tilde{d})$ admits at least one solution in the sense of Definition 3.2 (see [8]).

Step 2: The regularized problem corresponding to $S_{f, d}^{\rho}$. For any $\epsilon>0$, we denote by $\rho_{\epsilon}$ the Yosida regularization of $\rho$. 
Now, we set $\tilde{a}_{i}(x, \xi)=a_{i}(x, \xi) \chi_{\Omega}(x)+\frac{1}{\epsilon^{p_{i}(x)}}|\xi|^{p_{i}(x)-2} \xi \chi_{\tilde{\Omega} \backslash \Omega}(x)$ for all $(x, \xi) \in \tilde{\Omega} \times \mathbb{R}^{N}$, $\tilde{\rho}_{\epsilon}(s)=\frac{1}{\left|\tilde{\Gamma}_{N e}\right|} \rho_{\epsilon}(s)$ for all $s \in \mathbb{R}$. We consider the following problem $P_{\epsilon}(\tilde{\rho} \epsilon, \tilde{f}, \tilde{d})$

$$
\left\{\begin{array}{l}
-\sum_{i=1}^{N} \frac{\partial}{\partial x_{i}}\left(a_{i}\left(x, \frac{\partial}{\partial x_{i}} u_{\epsilon}\right) \chi_{\Omega}(x)+\frac{1}{\epsilon^{p_{i}(x)}}\left|\frac{\partial}{\partial x_{i}} u_{\epsilon}\right|^{p_{i}(x)-2} \frac{\partial}{\partial x_{i}} u_{\epsilon} \chi_{\tilde{\Omega} \backslash \Omega}(x)\right)+\left|u_{\epsilon}\right|^{P_{M}(x)-2} u_{\epsilon} \chi_{\Omega}=\tilde{f} \\
u_{\epsilon}=0 \\
\tilde{\rho}_{\epsilon}\left(u_{\epsilon}\right)+\sum_{i=1}^{N} \tilde{a}_{i}\left(x, \frac{\partial}{\partial x_{i}} u_{\epsilon}\right) \eta_{i}=\tilde{d}
\end{array}\right.
$$

$P_{\epsilon}\left(\tilde{\rho}_{\epsilon}, \tilde{f}, \tilde{d}\right)$ has at least one solution (see [8] ). So, there exists at least one measurable function $u_{\epsilon}: \tilde{\Omega} \rightarrow \mathbb{R}$ such that

$$
\left\{\begin{array}{l}
\sum_{i=1}^{N} \int_{\Omega}\left(a_{i}\left(x, \frac{\partial}{\partial x_{i}} u_{\epsilon}\right) \frac{\partial}{\partial x_{i}} \tilde{\varphi}\right) d x+\sum_{i=1}^{N} \int_{\tilde{\Omega} \backslash \Omega}\left(\frac{1}{\epsilon^{p_{i}(x)}}\left|\frac{\partial}{\partial x_{i}} u_{\epsilon}\right|^{p_{i}(x)-2} \frac{\partial}{\partial x_{i}} u_{\epsilon} \frac{\partial}{\partial x_{i}} \tilde{\varphi}\right) d x \\
\int_{\Omega}\left|u_{\epsilon}\right|^{P_{M}(x)-2} u_{\epsilon} \tilde{\varphi}=\int_{\Omega} f \tilde{\varphi} d x+\int_{\tilde{\Gamma}_{N e}}\left(\tilde{d}-\tilde{\rho}_{\epsilon}\left(u_{\epsilon}\right)\right) \tilde{\varphi} d \sigma,
\end{array}\right.
$$

where $u_{\epsilon} \in W_{D}^{1, \vec{p}(\cdot)}(\tilde{\Omega})$ and for all $\tilde{\varphi} \in W_{D}^{1, \vec{p}(.)}(\tilde{\Omega}) \cap L^{\infty}(\Omega)$.

Moreover, we have

$$
\left\{\begin{array}{l}
\tilde{\rho}_{\epsilon}\left(u_{\epsilon}\right) \mid \leq k_{3}:=\max \left\{\|\tilde{d}\|_{\infty},\left(\tilde{\rho}_{\epsilon} \circ b^{-1}\right)\left(\|f\|_{\infty}\right)\right\} \text { a.e. on } \tilde{\Gamma}_{N e} \\
\left|b\left(u_{\epsilon}\right)\right| \leq k_{4}:=\max \left\{\|f\|_{\infty} ;\left(b \circ \rho_{\epsilon}^{-1}\right)\left(\left|\tilde{\Gamma}_{N e}\right|\|\tilde{d}\|_{\infty}\right)\right\} \text { a.e. in } \Omega .
\end{array}\right.
$$

The following result gives a priori estimates on the solution $u_{\epsilon}$ of the problem $P_{\epsilon}\left(\tilde{\rho}_{\epsilon}, \tilde{f}, \tilde{d}\right)$ (see $[1,5])$.

Proposition 3.1. Let $u_{\epsilon}$ be a solution of the problem $P_{\epsilon}\left(\tilde{\rho}_{\epsilon}, \tilde{f}, \tilde{d}\right)$. Then, the following statements hold.

(i) There exists $C$ a positive constant independent of $\epsilon$ such that

$$
\sum_{i=1}^{N} \int_{\Omega}\left(\frac{\partial}{\partial x_{i}}\left|u_{\epsilon}\right|\right)^{p_{i}(x)} d x+\sum_{i=1}^{N} \int_{\tilde{\Omega} \backslash \Omega}\left(\frac{1}{\epsilon}\left|\frac{\partial}{\partial x_{i}} u_{\epsilon}\right|\right)^{p_{i}(x)} d x \leq C\left(\|\tilde{d}\|_{L^{1}\left(\tilde{\Gamma}_{N e}\right)}+\|f\|_{L^{1}(\Omega)}\right) .
$$

(ii)

$$
\int_{\Omega}\left|u_{\epsilon}\right|^{P_{M}(x)-1} d x+\int_{\tilde{\Gamma}_{N e}}\left|\tilde{\rho}_{\epsilon}\left(u_{\epsilon}\right)\right| d x \leq\left(\|\tilde{d}\|_{L^{1}\left(\tilde{\Gamma}_{N e}\right)}+\|f\|_{L^{1}(\Omega)}\right) .
$$

The following result states useful convergences results (see $[1,5])$.

Proposition 3.2. As $\epsilon \rightarrow 0$ we have

(i) $u_{\epsilon} \rightarrow u$ a.e. in $\Omega$ and a.e. on $\tilde{\Gamma}_{N e}$ with $u \in W_{D}^{1,\left(p_{1}^{-}, \ldots, p_{N}^{-}\right)}(\tilde{\Omega})$;

(ii) for all $i=1, \ldots N, \frac{\partial u_{\epsilon}}{\partial x_{i}} \rightarrow \frac{\partial u}{\partial x_{i}}=0$ in $L^{p_{i}^{-}}(\tilde{\Omega} \backslash \Omega)$ with $\frac{\partial u}{\partial x_{i}}=0$ in $\tilde{\Omega} \backslash \Omega$;

(iii) $a_{i}\left(x, \frac{\partial u_{\epsilon}}{\partial x_{i}}\right) \rightarrow a_{i}\left(x, \frac{\partial u}{\partial x_{i}}\right)$ weakly in $L^{1}(\Omega)$ and a.e.in $\Omega$.

Step 3: Proof of Theorem 2.1. Thanks to Proposition 3.2,

$\forall i=1, \ldots, N, \frac{\partial u}{\partial x_{i}}=0$ in $\tilde{\Omega} \backslash \Omega$, then

$u=$ constant a.e. 
on $\tilde{\Omega} \backslash \Omega$ so that, we conclude that $u \in W_{N e}^{1, \vec{p}(.)}(\Omega)$.

To show that $u$ is a solution of $P(\rho, f, d)$, we only have to prove the equality (2.9). The sequences $\left(\tilde{\rho}_{\epsilon}\left(u_{\epsilon}\right)\right)_{\epsilon>0}$ is uniformly bounded in $L^{\infty}\left(\tilde{\Gamma}_{N e}\right)$. Hence, there exists $v_{1} \in$ $L^{\infty}\left(\tilde{\Gamma}_{N e}\right)$ such that, as $\epsilon \rightarrow 0$,

$$
\tilde{\rho}_{\epsilon}\left(u_{\epsilon}\right) \rightarrow^{*} v_{1} \text { in } L^{\infty}\left(\tilde{\Gamma}_{N e}\right) .
$$

Let $\varphi \in W_{D}^{1, \vec{p}(.)}(\Omega) \cap L^{\infty}(\Omega)$. we consider the function $\varphi_{1} \in W_{D}^{1, \vec{p}(.)}(\tilde{\Omega}) \cap L^{\infty}(\Omega)$, such that

$$
\varphi_{1}=\varphi \chi_{\Omega}+\varphi_{N} \chi_{\tilde{\Omega} \backslash \Omega} .
$$

Then, $\varphi_{1}=$ constant on $\tilde{\Omega} \backslash \Omega$. Such function $\varphi_{1}$ in the equality (3.16) gives us

$$
\sum_{i=1}^{N} \int_{\Omega}\left(a_{i}\left(x, \frac{\partial}{\partial x_{i}} u_{\epsilon}\right) \cdot \frac{\partial}{\partial x_{i}} \varphi\right) d x+\int_{\Omega}\left|u_{\epsilon}\right|^{P_{M}(x)-2} u_{\epsilon} \varphi d x=\int_{\Omega} f \varphi d x+\left(d-\int_{\tilde{\Gamma}_{N e}} \tilde{\rho}_{\epsilon}\left(u_{\epsilon}\right) d \sigma\right) \varphi_{N} .
$$

Passing to the limit in (3.19) as $\epsilon \rightarrow 0$ and using the convergences in Proposition 3.2, one has

$\sum_{i=1}^{N} \int_{\Omega}\left(a_{i}\left(x, \frac{\partial}{\partial x_{i}} u\right) \frac{\partial}{\partial x_{i}} \varphi\right) d x+\lim _{\epsilon \rightarrow 0} \int_{\Omega} b\left(u_{\epsilon}\right) \varphi=\int_{\Omega} f \varphi d x+d \varphi_{N}-\left(\lim _{\epsilon \rightarrow 0} \int_{\tilde{\Gamma}_{N e}} \tilde{\rho}_{\epsilon}\left(u_{\epsilon}\right) d \sigma\right) \varphi_{N}$

By Proposition 3.2 and Lebesgue dominated convergence theorem, we deduce that

$$
b\left(u_{\epsilon}\right) \rightarrow b(u) \text { in } L^{1}(\Omega) .
$$

Thanks to (3.18) and (3.20), we deduce that

$$
\sum_{i=1}^{N} \int_{\Omega}\left(a_{i}\left(x, \frac{\partial}{\partial x_{i}} u\right) \frac{\partial}{\partial x_{i}} \varphi\right) d x+\int_{\Omega} b(u) \varphi=\int_{\Omega} f \varphi d x+d \varphi_{N}-\left(\int_{\tilde{\Gamma}_{N e}} v_{1} d \sigma\right) \varphi_{N} .
$$

We consider $w=b(u) \in L^{1}(\Omega)$ and $v=\int_{\tilde{\Gamma}_{N e}} v_{1} d \sigma \in \mathbb{R}$ to obtain from the above equality

$$
\sum_{i=1}^{N} \int_{\Omega}\left(a_{i}\left(x, \frac{\partial}{\partial x_{i}} u\right) \cdot \frac{\partial}{\partial x_{i}} \varphi\right) d x+\int_{\Omega} w \varphi d x=\int_{\Omega} f \varphi d x+(d-v) \varphi_{N} .
$$

To conclude that $(u, v)$ is a solution of $S_{f, d}^{\rho}$, it remain to show that

$$
v \in \rho\left(u_{N}\right) \text {. }
$$

We have $\tilde{\rho}_{\epsilon}\left(u_{\epsilon}\right) \rightarrow^{*} v_{1}$ in $L^{\infty}\left(\tilde{\Gamma}_{N e}\right)$ as $\epsilon \rightarrow 0$. So $\tilde{\rho}_{\epsilon}\left(u_{\epsilon}\right) \rightarrow v_{1}$ in $L^{p_{m}^{-}}\left(\tilde{\Gamma}_{N e}\right)$ as $\epsilon \rightarrow 0$.

We also have $u_{\epsilon} \rightarrow u$ in $L^{p_{m}^{-}}\left(\tilde{\Gamma}_{N e}\right)$ as $\epsilon \rightarrow 0$ and $\tilde{\rho}_{\epsilon} \rightarrow \frac{1}{\left|\tilde{\Gamma}_{N e}\right|} \rho$ in the sense of graph. Then (see [2]), $v_{1} \in \frac{1}{\left|\tilde{\Gamma}_{N e}\right|} \rho(u)$ a.e. on $\tilde{\Gamma}_{N e}$ and $v_{2}=\left|\tilde{\Gamma}_{N e}\right| v_{1} \in \rho(u)$ a.e. on $\tilde{\Gamma}_{N e}$.

We know that $u \equiv$ constant in $\tilde{\Omega} \backslash \Omega$ so $u \equiv$ constant on $\tilde{\Gamma}_{N e}$ and we get $v_{2} \in \rho\left(u_{N}\right)$.

Using the fact that $\mathcal{D}(\rho)=\mathbb{R}$ either $\rho\left(u_{N}\right)=s$ or $\rho\left(u_{N}\right)=[r, s]$ with $(r, s) \in \mathbb{R}^{2}$ such that $r<s$, it yields that $\frac{1}{\tilde{\Gamma}_{N e}} \int_{\tilde{\Gamma}_{N e}} v_{2} d \sigma \in \rho\left(u_{N}\right)$ and $v \in \rho\left(u_{N}\right) . \quad \square$ Let us prove now the inequality (2.10) of Theorem 2.1.

Proof. We have

$$
\left\{\begin{array}{l}
w_{1}=b\left(u_{1}\right), w_{2}=b\left(u_{2}\right) \\
v_{1} \in \rho\left(\left(u_{1}\right)_{N e}\right), v_{2} \in \rho\left(\left(u_{2}\right)_{N e}\right),
\end{array}\right.
$$


and for any $\varphi \in W_{N e}^{1, \vec{p}(.)}(\Omega) \cap L^{\infty}(\Omega)$,

$$
\int_{\Omega}\left(\sum_{i=1}^{N} a_{i}\left(x, \frac{\partial}{\partial x_{i}} u_{1}\right) \frac{\partial}{\partial x_{i}} \varphi\right) d x+\int_{\Omega} w_{1} \varphi d x=\int_{\Omega} f_{1} \varphi d x+\left(d_{1}-v_{1}\right) \varphi_{N e}
$$

and

$$
\int_{\Omega}\left(\sum_{i=1}^{N} a_{i}\left(x, \frac{\partial}{\partial x_{i}} u_{2}\right) \frac{\partial}{\partial x_{i}} \varphi\right) d x+\int_{\Omega} w_{2} \varphi d x=\int_{\Omega} f_{2} \varphi d x+\left(d_{2}-v_{2}\right) \varphi_{N e} .
$$

Subtracting (3.21) from (3.22), one has

$$
\left\{\begin{array}{l}
\int_{\Omega} \sum_{i=1}^{N}\left(a_{i}\left(x, \frac{\partial}{\partial x_{i}} u_{1}\right)-a_{i}\left(x, \frac{\partial}{\partial x_{i}} u_{2}\right)\right) \frac{\partial}{\partial x_{i}} \varphi d x+\int_{\Omega}\left(w_{1}-w_{2}\right) \varphi d x \\
+\left(v_{1}-v_{2}\right) \varphi_{N e}=\int_{\Omega}\left(f_{1}-f_{2}\right) \varphi d x+\left(d_{1}-d_{2}\right) \varphi_{N e} .
\end{array}\right.
$$

In (3.23) we take $\varphi=H_{\epsilon}\left(u_{1}-u_{2}+\epsilon \xi\right)$ where $\xi$ is any function in $W^{1, \vec{p}(.)}(\Omega)$. After calculus we obtain

$$
\left\{\begin{array}{l}
\int_{\Omega}\left(w_{1}-w_{2}\right) \xi \chi_{\left[u_{1}=u_{2}\right]} d x+\int_{\Omega}\left(w_{1}-w_{2}\right) \operatorname{sign}_{0}^{+} \chi_{\left[u_{1} \neq u_{2}\right]} d x+\left(v_{1}-v_{2}\right)\left(\xi_{N e}\right) \chi_{\left[\left(u_{1}\right)_{N e}=\left(u_{2}\right)_{N e}\right]} \\
+\left(v_{1}-v_{2}\right) \operatorname{sign}_{0}^{+}\left(\left(u_{1}\right)_{N e}-\left(u_{2}\right)_{N e}\right) \chi_{\left[\left(u_{1}\right)_{N e} \neq\left(u_{2}\right)_{N e}\right]} \leq \int_{\Omega}\left(f_{1}-f_{2}\right)^{+} d x+\left(d_{1}-d_{2}\right)^{+} .
\end{array}\right.
$$

Now, we consider the function $\xi_{0}$ defined as follows.

$$
\xi_{0}= \begin{cases}\operatorname{sign}_{0}^{+}\left(w_{1}-w_{2}\right) & \text { in }\left[u_{1}=u_{2}\right] \\ \operatorname{sign}_{0}^{+}\left(v_{1}-v_{2}\right) & \text { on } \Gamma_{N e} \\ 0 & \text { in } \mathbb{R}^{N} \backslash\left\{\left[u_{1}=u_{2}\right]\right\} .\end{cases}
$$

Replacing $\xi$ by $\xi_{0}$ in (3.24), we get

$$
\left\{\begin{array}{l}
\int_{\Omega}\left(w_{1}-w_{2}\right) \xi_{0} \chi_{\left[u_{1}=u_{2}\right]} d x+\int_{\Omega}\left(w_{1}-w_{2}\right) \operatorname{sign}_{0}^{+} \chi_{\left[u_{1} \neq u_{2}\right]} d x+\left(v_{1}-v_{2}\right)\left(\xi_{0}\right)_{N e} \chi_{\left[\left(u_{1}\right)_{N e}=\left(u_{2}\right)_{N e}\right]} \\
+\left(v_{1}-v_{2}\right) \operatorname{sign}_{0}^{+}\left(\left(u_{1}\right)_{N e}-\left(u_{2}\right)_{N e}\right) \chi_{\left[\left(u_{1}\right)_{N e} \neq\left(u_{2}\right)_{N e}\right]} \leq \int_{\Omega}\left(f_{1}-f_{2}\right)^{+} d x+\left(d_{1}-d_{2}\right)^{+} .
\end{array}\right.
$$

Taking into account the definition of $\xi_{0}$, one gets from (3.25)

$$
\left\{\begin{array}{l}
\int_{\Omega}\left(w_{1}-w_{2}\right) \operatorname{sign}_{0}^{+}\left(w_{1}-w_{2}\right) \chi_{\left[u_{1}=u_{2}\right]} d x+\int_{\Omega}\left(w_{1}-w_{2}\right) \operatorname{sign}_{0}^{+}\left(u_{1}-u_{2}\right) \chi_{\left[u_{1} \neq u_{2}\right]} d x \\
+\left(v_{1}-v_{2}\right) \operatorname{sign}_{0}^{+}\left(v_{1}-v_{2}\right) \chi_{\left[\left(u_{1}\right)_{N e}=\left(u_{2}\right)_{N e}\right]} \\
+\left(v_{1}-v_{2}\right) \operatorname{sign}_{0}^{+}\left(\left(u_{1}\right)_{N e}-\left(u_{2}\right)_{N e}\right) \chi_{\left[\left(u_{1}\right)_{N e} \neq\left(u_{2}\right)_{N e}\right]} \leq \int_{\Omega}\left(f_{1}-f_{2}\right)^{+} d x+\left(d_{1}-d_{2}\right)^{+}
\end{array}\right.
$$

which is equivalent to say

$$
\left\{\begin{array}{l}
\int_{\Omega}\left(w_{1}-w_{2}\right)^{+} \chi_{\left[u_{1}=u_{2}\right]} d x+\int_{\Omega}\left(w_{1}-w_{2}\right)^{+} \chi_{\left[u_{1} \neq u_{2}\right]} d x+\left(v_{1}-v_{2}\right)^{+} \chi_{\left[\left(u_{1}\right)_{N e}=\left(u_{2}\right)_{N e}\right]} \\
+\left(v_{1}-v_{2}\right)^{+} \chi_{\left[\left(u_{1}\right)_{N e} \neq\left(u_{2}\right)_{N e}\right]} \leq \int_{\Omega}\left(f_{1}-f_{2}\right)^{+} d x+\left(d_{1}-d_{2}\right)^{+}
\end{array}\right.
$$

and

$$
\left(v_{1}-v_{2}\right)^{+}+\int_{\Omega}\left(w_{1}-w_{2}\right)^{+} d x \leq \int_{\Omega}\left(f_{1}-f_{2}\right)^{+} d x+\left(d_{1}-d_{2}\right)^{+}
$$

which correspond to (2.10). 


\section{REFERENCES}

[1] Bonzi, B. K., Ouaro, S. and Zongo, F. D. Y., Entropy solution for nonlinear elliptic anisotropic homogeneous Neumann Problem, Int. J. Differ. Equ, Article ID 476781 (2013)

[2] Brezis, H., Opérateurs maximaux monotones et semigroupes de contractions dans les espaces de Hilbert, Amsterdam: North Holland, 1973

[3] Ding, Y., Ha-Duong, T., Giroire, J. and Moumas, V., Modeling of single-phase flow for horizontal wells in a stratified medium, Computers and Fluids, 33 (2004), No. 5-6, 715-727

[4] Giroire, J., Ha-Duong, T. and Moumas, V., A non-linear and non-local boundary condition for a diffusion equation in petroleum engineering, Math. Methods Appl. Sci., 28 (2005), No. 13, 1527-1552

[5] Ibrango, I. and Ouaro, S., Entropy solutions for anisotropic nonlinear Dirichlet problems, An. Univ. Craiova Ser. Mat. Inform., 42 (2015), No. 2, 347-364

[6] Fan, X. and Zhao, D., On the spaces $L^{p(.)}(\Omega)$ and $W^{m, p(.)}(\Omega)$. J. Math. Anal. Appl., 263 (2001), 424-446

[7] Fan, X., Anisotropic variable exponent Sobolev spaces and $\vec{p}($.$) -Laplacian equations, Complex Var. Elliptic Equ.,$ 56 (2011), No. 7-9, 623-642

[8] Ouaro, S. and Soma, S., Nonlinear elliptic problem involving non-local boundary conditions and variable exponent, Complex Var. Elliptic Equ., 63 (2018), No. 3, 437-461

UNIVERSITÉ JOSEPH KI-ZERBO

UFR. SCIENCES EXACTES ET APPLIQUÉES

LABORATOIRE DE MATHÉMATIQUES ET INFORMATIQUE (LAMI)

03 BP 7021 OUAGA 03, OUAGADOUGOU, BURKINA FASO

Email address: ouaro@yahoo.fr

Email address: kaboreadama59@yahoo.fr 